\title{
ON THE INTEGRAL REPRESENTATION OF POSITIVE LINEAR FUNCTIONALS
}

\author{
BY \\ A. E. NUSSBAUM( ${ }^{(1)}$
}

1. Introduction. Let $A$ be a $*$-algebra; i.e., $A$ is an algebra over the field of complex numbers with an involution-that is, a mapping $x \rightarrow x^{*}$ of $A$ onto $A$ such that $(x+y)^{*}=x^{*}+y^{*},(\alpha x)^{*}=\bar{\alpha} x^{*},(x y)^{*}=y^{*} x^{*},\left(x^{*}\right)^{*}=x$ for all $x$ and $y$ in $A$ and complex numbers $\alpha$. An element $x \in A$ is said to be selfadjoint if $x^{*}=x$. If $x \in A$, then $x=x_{1}+i x_{2}$, where $x_{1}=\left(\dot{x}+x^{*}\right) / 2$ and $x_{2}=\left(x-x^{*}\right) / 2 i . x_{1}$ and $x_{2}$ are selfadjoint elements of $A$ and are called the real and imaginary parts of $x$, respectively. We write $x_{1}=\operatorname{Re} x$ and $x_{2}=\operatorname{Im} x$. If $B$ is a subset of $A$ we denote by $B^{*}$ the set $\left\{x^{*} \mid x \in B\right\}$. A linear functional $f$ on $A$ is said to be positive if $f\left(x^{*} x\right) \geqq 0$ for all $x$ in $A$. A positive linear functional $f$ on a $*$-algebra $A$ is said to be real or hermitian if $f\left(x^{*}\right)=f(x)^{-}$for all $x$ in $A$. If $f$ is any positive linear functional on $A$, then $f\left(x^{*} y\right)=f\left(y^{*} x\right)^{-}$and $\left|f\left(x^{*} y\right)\right| \leqq f\left(x^{*} x\right)^{1 / 2} f\left(y^{*} y\right)^{1 / 2}$ (Schwarz's inequality) for all $x$ and $y$ in $A$. If $A$ has an identity $e$, we can take $y=e$ and obtain $f\left(x^{*}\right)=f(x)^{-}$and $|f(x)|^{2} \leqq M f\left(x^{*} x\right)$, where $M=f(e)$. A positive linear functional which satisfies these extra conditions (i.e., $f$ is real and $|f(x)|^{2} \leqq M f\left(x^{*} x\right)$ for all $x$ in $A$, where $M$ is a constant independent of $x$ ) is called extendible for reasons which the following proposition makes clear:

A necessary and sufficient condition that a positive linear functional $f$ on a *-algebra $A$ without identity can be extended so as to remain positive when an identity is added to $A$ is that $f$ be extendible in the above sense (cf. [8, p. 96], [9], and [12]).

Let $f$ be a positive linear functional on $A$. The elements $x$ in $A$ such that $f\left(x^{*} x\right)=0$ form a left ideal $I_{f}$ in $A$. If $x$ is an element in $A$ we denote by $x_{f}$ the coset of $A / I_{f}=H_{f}^{\prime}$ which contains $x$ and we define by

$$
\left(x_{f} \mid y_{f}\right)=f\left(y^{*} x\right)
$$

an inner product on $H_{f}^{\prime}$. Thus $H_{f}^{\prime}$ becomes a pre-Hilbert space. Let $H_{f}$ be the Hilbert space which is the completion of $H_{f}^{\prime}$. If $x \in A$ we denote by $U_{x}$ the operator in $H_{f}$ whose domain $D\left(U_{x}\right)=H_{f}^{\prime}$ and which maps $y_{f}$ into $(x y)_{f}$. Then $U_{x}$ is a densely defined operator in $H_{f}$ and $U_{x^{*}} \subset U_{x}^{*}$ (i.e., the adjoint of $U_{x}$ is an extension of $U_{x^{*}}$ ). Hence $U_{x^{*}}$ has a closure [ $U_{x^{*}}$ ] for every $x$ in $A$. Furthermore $U_{x y}=U_{x} U_{y}$, $U_{\alpha x}=\alpha U_{x}$, and $U_{x+y}=U_{x}+U_{y}$ for all $x$ and $y$ in $A$ and complex numbers $\alpha$.

Received by the editors October 6, 1966.

( ${ }^{1}$ This work was in part supported by National Science Foundation Grant No. GP-3583. 
Clearly, the necessary and sufficient condition that $U_{x}$, and hence $\left[U_{x}\right]$, be a bounded operator is that there exists a constant $M_{x}$ such that

$$
f\left(y^{*} x^{*} x y\right) \leqq M_{x} f\left(y^{*} y\right) \text { for all } y \text { in } A .
$$

If $U_{x}$ is bounded for every $x \in A$, then $f$ is said to be unitary (cf. [6] and [8]). If $f$ is unitary, then $D\left(\left[U_{x}\right]\right)=H_{f}$ for every $x \in A$ and $x \rightarrow T_{x}=\left[U_{x}\right]$ is a $*$-representation of $A$ by bounded operators on $H_{f}$ and $f\left(x y z^{*}\right)=\left(T_{x} y_{f} \mid z_{f}\right)$. A *-homomorphism of $A$ onto the field $C$ of complex numbers is called a unitary character of $A$. Thus a homomorphism $\chi$ of $A$ onto $C$ is a unitary character of $A$ if and only if $\chi\left(x^{*}\right)=\chi(x)^{-}$ for all $x \in A$. If $A$ is a commutative $*$-algebra, we denote by $\hat{A}$ the set of unitary characters of $A$ together with the weakest topology such that the mappings $\hat{x}: \chi \rightarrow \chi(x), x \in A$, are continuous. Clearly $\hat{A}$ is a Hausdorff space. Suppose now that $f$ is a unitary positive linear functional on a commutative $*$-algebra $A$. Let $R$ be the $C^{*}$-algebra generated by $\left\{T_{x}\right\}, x \in A$. Using the spectral theorem of the commutative $C^{*}$-algebra $R, \mathrm{R}$. Godement has obtained the following integral representation for $f$, which he has called the Plancherel formula for $f$ :

THEOREM 1 (R. Godement [6, p. 76]). Let $f$ be a positive linear functional on a commutative *-algebra $A$. If $f$ is unitary, then there exists a positive Radon measure $\mu_{f}$ on a locally compact subset $\sigma_{f}$ of $\hat{A}$ such that

(a) $\hat{x}(\chi)=\chi(x)$ belongs to $L^{2}\left(\mu_{f}\right)$ for every $x \in A$;

(b) $f(x y z)=\int_{\sigma_{f}} \chi(x y z) d \mu_{f}(\chi)$ for all $x, y$, and $z$ in $A$.

If, furthermore, $f$ is extendable, then $\mu_{f}$ is a finite measure and

$$
f(x)=\int_{\sigma_{f}} \chi(x) d \mu_{f}(\chi)
$$

for all $x$ in $A$.

(Godement assumes in his definition of a positive linear functional that the functional is real. This condition is not necessary, however, for the proof of (b).)

According to $\mathrm{R}$. Godement the extension of Theorem 1 to arbitrary positive linear functionals is of fundamental importance (cf. loc. cit. p. 78). It follows, however, from the results of R. B. Zarhina [13] on the two-dimensional moment problem that Godement's Plancherel formula is not valid for an arbitrary positive linear functional on an arbitrary commutative $*$-algebra. It is not valid, for example, for every positive linear functional on the $*$-algebra of polynomials in two variables (cf. [7, pp. 232-236]). On the other hand there exist positive linear functionals for which Plancherel's formula ((b) of Theorem 1) holds, but which are not unitary. For example, if $A$ is the commutative $*$-algebra of complex polynomials $p(t)$ with respect to the ordinary operations of addition and multiplication and involution $p^{*}(t)=p(t)^{-}$and

$$
f(p)=\int_{-\infty}^{\infty} p(t) e^{-|t|} d t
$$


for $p \in A$, then $f$ is a positive linear functional on $A$ which by definition has an integral representation of the form (b). But $f$ is not unitary, for otherwise there exists a constant $M$ such that

$$
\int_{-\infty}^{\infty} t^{2} t^{2 n} e^{-|t|} d t \leqq M \int_{-\infty}^{\infty} t^{2 n} e^{-|t|} d t
$$

for all $n \geqq 0$. This inequality is obviously false, for the left-hand side is equal to $2 \Gamma(2 n+3)=2(2 n+2)$ ! and the right-hand side is equal to $2 M \Gamma(2 n+1)=2 M(2 n)$ !

The main purpose of this paper is to extend Theorem 1 to positive linear functionals which satisfy certain growth conditions, but which are not necessarily unitary.

We say that a positive linear functional $f$ on a commutative $*$-algebra $A$ is quasi-unitary if there exists a subset $A_{0}$ of $A$ such that

$$
\sum_{n=1}^{\infty} f\left(\left(x x^{*}\right)^{n}\right)^{-1 / 2 n}=\infty \text { for all } x \in A_{0}
$$

and if for every $x \in A$ there exists an element $y$ in the $*$-algebra $A_{e}$ obtained from $A$ by adjoining an identity element $e$ (if $A$ does not have an identity element) which is a polynomial with complex coefficients in finitely many elements of $A_{0} \cup A_{0}^{*}$ such that

$$
f\left(x x^{*} z z^{*}\right) \leqq f\left(y y^{*} z z^{*}\right) \text { for all } z \in A .
$$

(Note that condition (2) is automatically satisfied if (1) holds for all $x \in A$.)

In $\$ 2$ we show that if $f$ is a quasi-unitary positive linear functional on a commutative $*$-algebra $A$, then $x \rightarrow T_{x}=\left[U_{x}\right]$ is a $*$-representation of $A$ by permuting (in general unbounded) normal operators, and if $f$ is unitary, it is a fortiori quasiunitary. (For a precise definition of a $*$-representation of $A$ by unbounded normal operators cf. Theorem 2.)

The main result of this paper is Theorem 4 of $\S 3$ which states that Godement's theorem remains true mutatis mutandis if unitary is replaced by quasi-unitary and, in addition, the positive linear functional $f$ satisfies the following separability condition (d):

There exists a countable subset $D$ of $A_{e}$ such that for every $x \in A$ there exists a $y \in A_{e}$ which is a polynomial with complex coefficients in finitely many elements of $D$ such that

$$
f\left(x x^{*} z z^{*}\right) \leqq f\left(y y^{*} z z^{*}\right) \text { for all } z \in A .
$$

This condition is satisfied if $f$ is unitary, if we take for $D=\{e\}$.

Thus Theorem 4 includes Godement's theorem as a special case, but it also yields the integral representation of the nonunitary positive linear functional of the example given above. Other examples should not be difficult to construct. 
2. *-representation. Let $A$ be a commutative $*$-algebra and $f$ a positive linear functional on $A$. We denote by $A_{e}$ the *-algebra obtained from $A$ by adjoining an identity element $e$ to $A$, if $A$ does not have an identity element. If $A$ does have an identity element, we set $A_{e}=A$. Let $T_{x}=\left[U_{x}\right]$, where $U_{x}$ is the operator in $H_{f}$ defined in the introduction. Then $f\left(x y z^{*}\right)=\left(T_{x} y_{f} \mid z_{f}\right)$ for all $x, y$, and $z$ in $A$.

LEMMA 1.

$$
\sum_{n=1}^{\infty} f\left(\left(x x^{*}\right)^{n}\right)^{-1 / 2 n}=\infty \Leftrightarrow \sum_{n=1}^{\infty} f\left(\left(x x^{*}\right)^{2 n}\right)^{-1 / 4 n}=\infty .
$$

Proof. That $\sum_{n=1}^{\infty} f\left(\left(x x^{*}\right)^{2 n}\right)^{-1 / 4 n}=\infty$ implies $\sum_{n=1}^{\infty} f\left(\left(x x^{*}\right)^{n}\right)^{-1 / 2 n}=\infty$ is obvious. To prove the reverse implication we may assume without loss of generality that $f\left(x x^{*}\right)=1$, for if $f\left(x x^{*}\right)=0$ then $f\left(\left(x x^{*}\right)^{n}\right)=0$ for all $n \geqq 1$ by Schwarz's inequality. We assume, therefore, that $f\left(x x^{*}\right)=1$. Then $f\left(\left(x x^{*}\right)^{n+1}\right)^{1 / 2 n}$ is a nondecreasing function of $n \geqq 1$. Indeed,

$$
f\left(\left(x x^{*}\right)^{2}\right)=f\left(x\left(x^{*} x x^{*}\right)\right) \leqq f\left(x x^{*}\right)^{1 / 2} f\left(\left(x x^{*}\right)^{3}\right)^{1 / 2}=f\left(\left(x x^{*}\right)^{3}\right)^{1 / 2} .
$$

Hence

$$
f\left(\left(x x^{*}\right)^{2}\right)^{1 / 2} \leqq f\left(\left(x x^{*}\right)^{3}\right)^{1 / 4} .
$$

Assume now that $f\left(\left(x x^{*}\right)^{n+1}\right)^{1 / 2 n} \leqq f\left(\left(x x^{*}\right)^{n+2}\right)^{1 /(2 n+2)}$, then

Hence

$$
\begin{aligned}
f\left(\left(x x^{*}\right)^{n+2}\right) & =f\left(x^{n+1}\left(x x^{* n+2}\right)\right) \leqq f\left(\left(x x^{*}\right)^{n+1}\right)^{1 / 2} f\left(\left(x x^{*}\right)^{n+3}\right)^{1 / 2} \\
& \leqq f\left(\left(x x^{*}\right)^{n+2}\right)^{n /(2 n+2)} f\left(\left(x x^{*}\right)^{n+3}\right)^{1 / 2}
\end{aligned}
$$

$$
f\left(\left(x x^{*}\right)^{n+2}\right)^{(n+2) /(2 n+2)} \leqq f\left(\left(x x^{*}\right)^{n+3}\right)^{1 / 2}
$$

and therefore $f\left(\left(x x^{*}\right)^{n+2}\right)^{1 /(2 n+2)} \leqq f\left(\left(x x^{*}\right)^{n+3}\right)^{1 /(2 n+4)}$. Hence, by finite induction, $f\left(\left(x x^{*}\right)^{n+1}\right)^{1 / 2 n}$ is a nondecreasing function of $n$. It follows that

$$
\sum_{n=1}^{\infty} f\left(\left(x x^{*}\right)^{n+1}\right)^{-1 / 2 n}=\infty \Rightarrow \sum_{n=1}^{\infty} f\left(\left(x x^{*}\right)^{2 n}\right)^{-1 /(4 n-2)}=\infty
$$

and hence

$$
\sum_{n=1}^{\infty} f\left(\left(x x^{*}\right)^{n+1}\right)^{-1 / 2 n}=\infty \Rightarrow \sum_{n=1}^{\infty} f\left(\left(x x^{*}\right)^{2 n}\right)^{-1 / 4 n}=\infty .
$$

Now, if $M_{n}>0$ for $n \geqq 1$ and if $p$ is an arbitrary but fixed real number, then $\sum_{n=1}^{\infty}\left(M_{n}\right)^{-1 /(n+p)}$ converges if and only if $\sum_{n=1}^{\infty}\left(M_{n}\right)^{-1 / n}$ converges (cf. [3, p. 106]). Hence

$$
\sum_{n=1}^{\infty} f\left(\left(x x^{*}\right)^{n+1}\right)^{-1 / 2 n}=\infty \Leftrightarrow \sum_{n=1}^{\infty} f\left(\left(x x^{*}\right)^{n}\right)^{-1 / 2 n}=\infty
$$

and therefore

$$
\sum_{n=1}^{\infty} f\left(\left(x x^{*}\right)^{n}\right)^{-1 / 2 n}=\infty \Rightarrow \sum_{n=1}^{\infty} f\left(\left(x x^{*}\right)^{2 n}\right)^{-1 / 4 n}=\infty .
$$


THEOREM 2. Suppose there exists a subset $A_{0}$ of $A$ such that

1. $\sum_{n=1}^{\infty} f\left(\left(x x^{*}\right)^{n}\right)^{-1 / 2 n}=\infty$ for all $x \in A_{0}$;

2. for every $x \in A$ there exists an element $y \in A_{e}$ which is a polynomial with complex coefficients in finitely many elements of $A_{0} \cup A_{0}^{*}$ such that

$$
f\left(x x^{*} z z^{*}\right) \leqq f\left(y y^{*} z z^{*}\right) \text { for all } z \in A .
$$

Then $x \rightarrow T_{x}$ is a $*$-representation of $A$ by permuting (in general unbounded) normal operators. That is, $\left\{T_{x}\right\}, x \in A$, are permuting normal operators (i.e., their resolutions of the identity permute), $T_{x+y}=\left[T_{x}+T_{y}\right], T_{x y}=\left[T_{x} T_{y}\right], T_{\alpha x}=\left[\alpha T_{x}\right]$ and $T_{x^{*}}=T_{x}^{*}$ for all $x, y \in A$ and complex numbers $\alpha$.

Proof. We first observe that if condition 1 holds for a given $x$, then it also holds for $x_{1}=\operatorname{Re} x$ and $x_{2}=\operatorname{Im} x$ since

$$
f\left(\left(x x^{*}\right)^{n}\right)=\sum_{k=0}^{n}\left(\begin{array}{l}
n \\
k
\end{array}\right) f\left(x_{1}^{2 k} x_{2}^{2(n-k)}\right)
$$

and hence

$$
f\left(\left(x x^{*}\right)^{n}\right) \geqq f\left(x_{1}^{2 n}\right) \text { and } f\left(\left(x x^{*}\right)^{n}\right) \geqq f\left(x_{2}^{2 n}\right) \text { for all } n \geqq 1 .
$$

Since $U_{x^{*}} \subset U_{x}^{*}$, it follows that $T_{x^{*}} \subset T_{x}^{*}$ for every $x \in A$. Hence, if $x$ is selfadjoint, $T_{x}$ is a closed symmetric operator. To prove the theorem, it is sufficient to show that (i) $T_{x}$ is selfadjoint for every selfadjoint element $x$ in $A$ and (ii) $T_{x}$ and $T_{y}$ permute if $x$ and $y$ are any two selfadjoint elements of $A$. Indeed, suppose that (i) and (ii) hold. Let $x$ be any element in $A$. Then $U_{x x^{*}} \subset U_{x} U_{x^{*}} \subset T_{x} T_{x^{*}} \subset T_{x} T_{x}^{*}$. Hence $T_{x x^{*}}=U_{x x^{*}}^{*} \supset T_{x} T_{x}^{*}$, since $T_{x} T_{x}^{*}$ is selfadjoint. But this implies that $T_{x x^{*}}=T_{x} T_{x}^{*}$, since $T_{x x^{*}}$ is symmetric. Similarly $U_{x x^{*}}=U_{x^{*} x} \subset T_{x^{*}} T_{x} \subset T_{x}^{*} T_{x}$. Hence $T_{x x^{*}}=U_{x x^{*}}^{*}$ $\supset T_{x}^{*} T_{x}$ and therefore $T_{x x^{*}}=T_{x}^{*} T_{x}$. Hence $T_{x} T_{x}^{*}=T_{x}^{*} T_{x}$; i.e., $T_{x}$ is normal for every $x \in A$. Suppose $x$ and $y$ are any two elements in $A$. Write $x=x_{1}+i x_{2}$, $y=y_{1}+i y_{2}$, where $x_{1}, y_{1}$ and $x_{2}, y_{2}$ are the real and imaginary parts of $x$ and $y$, respectively. Now $U_{x}=U_{x_{1}}+i U_{x_{2}} \subset T_{x_{1}}+i T_{x_{2}}$ and $T_{x_{1}}$ and $T_{x_{2}}$ are permuting selfadjoint operators. Hence $T_{x_{1}}+i T_{x_{2}}$ is a normal operator and $T_{x}=\left[U_{x}\right] \subset T_{x_{1}}+i T_{x_{2}}$. But $T_{x}$ is normal as we have seen. Hence $T_{x}=T_{x_{1}}+i T_{x_{2}}$. Similarly $T_{y}=Y_{y_{1}}+i T_{y_{2}}$. But $T_{x_{1}}, T_{x_{2}}, T_{y_{1}}, T_{y_{2}}$ are permuting selfadjoint operators by (ii). Hence $T_{x}$ and $T_{y}$ permute. Moreover, $T_{\alpha x}=\left[\alpha T_{x}\right]$ for $U_{\alpha x}=\alpha U_{x}$ and hence $T_{x^{*}}=T_{x_{1}}+i T_{-x_{2}}$ $=T_{x_{1}}-i T_{x_{2}}=T_{x}^{*}$. Now, $T_{x}+T_{y}=\left(T_{x_{1}}+i T_{x_{2}}\right)+\left(T_{y_{1}}+i T_{y_{2}}\right)=\left(T_{x_{1}}+T_{y_{1}}\right)+i\left(T_{x_{2}}+T_{y_{2}}\right)$ $\subset T_{x_{1}+y_{1}}+i T_{x_{2}+y_{2}}=T_{x+y}$, for $\left[T_{x_{1}}+T_{y_{1}}\right]=T_{x_{1}+y_{2}}$, and $\left[T_{x_{2}}+T_{y_{2}}\right]=T_{x_{2}+y_{2}}$. (Because $U_{x_{1}+y_{1}}=U_{x_{1}}+U_{y_{1}} \subset T_{x_{1}}+T_{y_{1}}$ and hence taking adjoints: $T_{x_{1}+y_{1}}$ $\supset T_{x_{1}}+T_{y_{1}}$. But $\left[T_{x_{1}}+T_{y_{1}}\right]$ is selfadjoint by the operational calculus for normal operators. Hence $\left[T_{x_{1}}+T_{y_{1}}\right]=T_{x_{1}+y_{1}}$. Similarly, $\left[T_{x_{2}}+T_{y_{2}}\right]=T_{x_{2}+y_{2}}$.) From $T_{x}+T_{y} \subset T_{x+y}$, and the fact that $T_{x}, T_{y}, T_{x+y}$ are normal and $T_{x}$ and $T_{y}$ permute, follows by the operational calculus for normal operators and the fact that a normal operator is maximal (in the sense that it does not have a proper normal extension) that $\left[T_{x}+T_{y}\right]=T_{x+y}$. Finally, $U_{x} U_{y}=U_{x_{1} y_{1}-x_{2} y_{2}}+i U_{x_{1} y_{2}+x_{2} y_{1}}$. Hence $\left[U_{x} U_{y}\right]$ 
$=T_{x_{1} y_{1}-x_{2} y_{2}}+i T_{x_{1} y_{2}+x_{2} y_{1}}=T_{x y}$. But $\quad\left[U_{x} U_{y}\right]=\left(U_{x} U_{y}\right)^{* *} \supset\left(U_{y}^{*} U_{x}^{*}\right)^{*} \supset U_{x}^{* *} U_{y}^{* *}$ $=T_{x} T_{y}$. Hence $T_{x} T_{y} \subset T_{x y}$. From this follows, since $T_{x}, T_{y}, T_{x y}$ are normal and $T_{x}$ and $T_{y}$ permute-as above-that $\left[T_{x} T_{y}\right]=T_{x y}$.

Let $x$ now be the real or imaginary part of an element of $A_{0}$ and $y$ any element of $A$, then

$$
\left\|T_{x}^{n} y_{f}\right\|^{2}=\left\|\left(x^{n} y\right)_{f}\right\|^{2}=f\left(x^{2 n} y y^{*}\right) \leqq f\left(x^{4 n}\right)^{1 / 2} f\left(\left(y y^{*}\right)^{2}\right)^{1 / 2}
$$

and hence

$$
\sum_{n=1}^{\infty}\left\|T_{x}^{n} y_{f}\right\|^{-1 / n} \geqq \sum_{n=1}^{\infty} f\left(x^{4 n}\right)^{-1 / 4 n} f\left(\left(y y^{*}\right)^{2}\right)^{-1 / 4 n} .
$$

But $\sum_{n=1}^{\infty} f\left(x^{2 n}\right)^{-1 / 2 n}=\infty$ by condition 1 and the above remark and hence $\sum_{n=1}^{\infty} f\left(x^{4 n}\right)^{-1 / 4 n}=\infty$ by Lemma 1 . Therefore

$$
\sum_{n=1}^{\infty}\left\|T_{x}^{n} y_{f}\right\|^{-1 / n}=\infty
$$

That is, every element of $H_{f}^{\prime}$ is a quasi-analytic vector for $T_{x}$ (for the theory of quasi-analytic vectors cf. [11]). Hence $T_{x}$ is selfadjoint for every $x$ which is the real or imaginary part of an element of $A_{0}$ by Theorem 2 of loc. cit. If $x$ and $y$ are the real or imaginary parts of any two elements of $A_{0}$, then $T_{x}$ and $T_{y}$ permute by Theorem 6 of loc. cit.

Next, let $x_{1}$ and $x_{2}$ be any two selfadjoint elements of $A$. Let $x=x_{1}+i x_{2}$ and choose, using condition 2 , an element $y \in A_{e}$ which is a polynomial in the elements $a_{1}, a_{2}, \ldots, a_{m}, a_{1}^{*}, a_{2}^{*}, \ldots, a_{m}^{*}$, where $a_{1}, a_{2}, \ldots, a_{m}$ are elements of $A_{0}$ such that

$$
f\left(x x^{*} z z^{*}\right) \leqq f\left(y y^{*} z z^{*}\right) \text { for all } z \in A .
$$

Replacing $a_{k}$ by $\operatorname{Re} a_{k}+i \operatorname{Im} a_{k}, k=1,2, \ldots, m$, we see that

$$
y=\sum c_{i_{1}} \cdots i_{n} y_{1}^{i_{1}} \cdots y_{n}^{i_{n}}
$$

where $y_{1}, \ldots, y_{n}$ are the real or imaginary parts of elements of $A_{0}$ and the $c_{i_{1}} \cdots i_{n}$ are complex numbers. We may assume that $y$ is selfadjoint, for otherwise replace $y$ by $y_{1}^{2}+y_{2}^{2}+e$, where $y_{1}=\operatorname{Re} y$ and $y_{2}=\operatorname{Im} y$. For, if $u=y_{1}^{2}+y_{2}^{2}$, then

$$
f\left(y y^{*} z z^{*}\right)=f\left(\left(y_{1}^{2}+y_{2}^{2}\right) z z^{*}\right) \leqq f\left(\left(u^{2}+2 u+e\right) z z^{*}\right)=f\left((u+e)^{2} z z^{*}\right) .
$$

Finally, we may assume that the coefficients $c_{i_{1}} \ldots i_{n}$ are real, for

$$
y=\frac{y+y^{*}}{2}=\frac{1}{2} \sum\left(c_{i_{1} \cdots i_{n}}+\bar{c}_{i_{1} \cdots i_{n}}\right) y_{1}^{i_{1}} \cdots y_{n}^{i_{n}}=\sum\left(\operatorname{Re} c_{i_{1}} \cdots i_{n}\right) y_{1}^{i_{1}} \cdots y_{n}^{i_{n}} .
$$

If $w \in A_{e}$, we denote by $U_{w}$ the operator $x \rightarrow(w y)_{f}$ in $H_{f}$ with domain $H_{f}^{\prime}$. Clearly

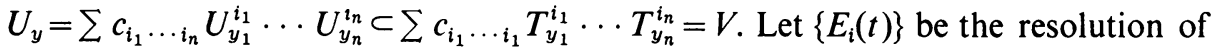
the identity of $T_{y_{i}}, i=1, \ldots, n$. Let $k_{i}$ be any nonnegative integer and $E_{i}^{\left(k_{i}\right)}=E_{i}\left(k_{i}\right)$ $-E_{i}\left(-k_{i}\right)$ and $E_{\left(k_{1}, \ldots, k_{n}\right)}=E_{n}^{\left(k_{1}\right)} \ldots E_{n}^{\left(k_{n}\right)} . E_{i}(t)$ permutes with $T_{x_{1}}, T_{x_{2}}, T_{y_{1}}, \ldots, T_{y_{n}}$ 
by Corollary 5 of [11]. Hence $E_{k}=E_{\left(k_{1}, \ldots, k_{n}\right)}$ permutes with $T_{x_{1}}, T_{x_{2}}, T_{y}=\left[U_{y}\right]$, $T_{y_{1}}, \ldots, T_{y_{n}}$ and hence with $V$. Now,

$$
E_{k} U_{k} \subset E_{k} V \subset \sum c_{i_{1} \cdots i_{n}}\left(T_{y_{1}} E_{1}^{\left(k_{1}\right)}\right)^{i_{1}} \cdots\left(T_{y_{n}} E_{n}^{\left(k_{n}\right)}\right)^{i_{n}} \subset V E_{k}
$$

and $T_{y_{i}} E_{i}^{\left(k_{i}\right)}$ is a bounded selfadjoint operator which permutes with $T_{y_{j}} E_{j}^{\left(k_{j}\right)}$, for $j=1, \ldots, n$. Hence $V E_{k}$ is a bounded selfadjoint operator and therefore

$$
T_{y} E_{k}=\left(E_{k} U_{y}\right)^{*} \supset V E_{k} .
$$

Hence $T_{y} E_{k}=V E_{k}$ and therefore $T_{y} E_{k}$ is a bounded selfadjoint operator.

Now,

$$
f\left(x x^{*} z z^{*}\right)=f\left(\left(x_{1}^{2}+x_{2}^{2}\right) z z^{*}\right)=\left\|T_{x_{1}} z_{f}\right\|^{2}+\left\|T_{x_{2}} z_{f}\right\|^{2} \leqq f\left(y y^{*} z z^{*}\right)=\left\|T_{y} z_{f}\right\|^{2} .
$$

That is, $\left\|T_{x_{1}} z_{f}\right\|^{2}+\left\|T_{x_{2}} z_{f}\right\|^{2} \leqq\left\|T_{y} z_{f}\right\|^{2}$ for all $z_{f} \in H_{f}^{\prime}$. It follows that $D\left(T_{y}\right) \subset D\left(T_{x_{1}}\right)$, $D\left(T_{y}\right) \subset D\left(T_{x_{2}}\right)$ and

$$
\left\|T_{x_{1}} u\right\|^{2}+\left\|T_{x_{2}} u\right\|^{2} \leqq\left\|T_{y} u\right\|^{2} \text { for all } u \in D\left(T_{y}\right) .
$$

Hence $\left\|T_{x_{1}} E_{k} u\right\|^{2}+\left\|T_{x_{2}} E_{k} u\right\|^{2} \leqq\left\|T_{y} E_{k} u\right\|^{2} \leqq\left\|T_{y} E_{k}\right\|^{2}\|u\|^{2}$ for all $u \in H_{f}$. Hence $T_{x_{1}} E_{k}$ and $T_{x_{2}} E_{k}$ are bounded. From this and the fact that $E_{k}$ permutes with $T_{x_{1}}$ and $T_{x_{2}}$ and $E_{k} \rightarrow I$ as $k_{1} \rightarrow \infty, \ldots, k_{n} \rightarrow \infty$, follows by standard Hilbert space methods that $T_{x_{1}}$ and $T_{x_{2}}$ are selfadjoint. It can also be seen as follows:

$$
\left\|T_{x_{1}}^{n} E_{k} u\right\|=\left\|\left(T_{x_{1}} E_{k}\right)^{n} u\right\| \leqq\left\|T_{x_{1}} E_{k}\right\|^{n}\|u\|
$$

and similarly

$$
\left\|T_{x_{2}}^{n} E_{k} u\right\| \leqq\left\|T_{x_{2}} E_{k}\right\|^{n}\|u\| \text { for all } u \in H_{f} \text { and all } k \text {. }
$$

Hence every vector of the set $D=\left\{E_{k} u \mid u \in H_{f}\right.$, all $\left.k\right\}$ is a quasi-analytic vector for $T_{x_{1}}$ and $T_{x_{2}}$, respectively. Since $D$ is dense in $H_{f}$ it follows from Theorem 2 of [11] that $T_{x_{1}}$ and $T_{x_{2}}$ are selfadjoint.

Finally,

$$
\left(E_{k} U_{x_{1}}^{m} U_{x_{2}}^{n}\right)^{*} \supset T_{x_{2}}^{n} T_{x_{1}}^{m} E_{k} \supset\left(T_{x_{2}} E_{k}\right)^{n}\left(T_{x_{1}} E_{k}\right)^{m}
$$

and therefore $\left(E_{k} U_{x_{1}^{m} x_{2}^{n}}\right)^{*}=T_{x_{2}}^{n} T_{x_{1}}^{m} E_{k}$. Similarly,

$$
\left(E_{k} U_{x_{1}^{m} x_{2}^{n}}\right)^{*}=\left(E_{k} U_{x_{2}^{n} x_{1}^{m}}\right)^{*}=T_{x_{1}}^{m} T_{x_{2}}^{n} E_{k} .
$$

Hence $T_{x_{1}}^{m} T_{x_{2}}^{n} E_{k}=T_{x_{2}}^{n} T_{x_{1}}^{m} E_{k}$ for all $n$ and $m \geqq 1$ and all $k$. Hence $T_{x_{1}}^{m} T_{x_{2}}^{n} u=T_{x_{2}}^{n} T_{x_{1}}^{m} u$ for all $u \in D$ and $n$ and $m \geqq 1$. Hence $T_{x_{1}}$ and $T_{x_{2}}$ permute by Theorem 6 of [11]. (That $T_{x_{1}}$ and $T_{x_{2}}$ permute follows also by standard Hilbert space techniques from the fact that $E_{k}$ reduces $T_{x_{1}}$ and $T_{x_{2}}$, respectively, to bounded permuting selfadjoint operators and the fact that $E_{k} \rightarrow I$ as $k_{1} \rightarrow \infty, \ldots, k_{n} \rightarrow \infty$.)

Definition 1. A positive linear functional f on a commutative *-algebra $A$ will be called quasi-unitary, if there exists a subset $A_{0}$ of As such that conditions 1 and 2 of Theorem 2 hold. 
PROPOSITION 1. Every unitary positive linear functional on a commutative $*$-algebra is quasi-unitary.

Proof. Let $f$ be a unitary positive linear functional on a commutative $*$-algebra $A$. Let $x$ be any element in $A$, then $f\left(\left(x x^{*}\right)^{n}\right) \leqq M_{x} f\left(\left(x x^{*}\right)^{n-1}\right)$ for all $n \geqq 2$ and hence

$$
f\left(\left(x x^{*}\right)^{n}\right) \leqq M_{x}^{n-1} f\left(x x^{*}\right) \text { for all } n \geqq 1 .
$$

Hence $\sum_{n=1}^{\infty} f\left(\left(x x^{*}\right)^{n}\right)^{-1 / 2 n}=\infty$. To satisfy conditions 1 and 2 of Theorem 2 we may therefore take $A_{0}=A$. However, it is sufficient to take $A_{0}=\left\{x_{0}\right\}$, where $x_{0}$ is an arbitrary element in $A$, for we may choose for $x \in A$ the element $y$ in condition 2 to be $M_{x}^{1 / 2} e$, which is a polynomial in $x_{0}$ and $x_{0}^{*}$.

The positive linear functional which we have considered in the introduction is quasi-unitary, but not unitary (as we have seen). Indeed, let $A_{0}=\{t\}$. Then

$$
f\left(t^{2 n}\right)=\int_{-\infty}^{\infty} t^{2 n} e^{-|t|} d t=2 \int_{0}^{\infty} t^{2 n} e^{-t} d t=2(2 n) !<2(2 n)^{2 n}
$$

that is, $f\left(t^{2 n}\right)<2(2 n)^{2 n}$ for all $n \geqq 1$. Hence $\sum_{n=1}^{\infty} f\left(t^{2 n}\right)^{-1 / 2 n}=\infty$. Condition 2 of Theorem 2 is obviously satisfied, for every element in $A$ is a polynomial in $t$.

3. Integral representation of quasi-unitary positive linear functionals. Let $f$ be a quasi-unitary positive linear functional on a commutative $*$-algebra $A$ and $x \rightarrow T_{x}$ the corresponding $*$-representation (cf. Theorem 2). Let $R$ be the bi-commutant of $\left\{T_{x} \mid x \in A\right\}$, then $R$ is the von Neumann algebra generated by the spectral projections of the normal operators $\left\{T_{x}\right\}, x \in A$. Let $T \rightarrow \hat{T}$ be the Gelfand representation of the $C^{*}$-algebra $R$ onto $C(\mathfrak{M})-\mathfrak{M}$ is the spectrum of $R$. Let $\bar{C}(\mathfrak{M})$ be the algebra of continuous functions on $\mathfrak{M}$ which are $\infty$ only on a nowhere dense set. (If $f$ and $g$ are elements of $\bar{C}(\mathfrak{M})$, then $f g$ and $f+g$ are defined to be the unique elements in $\bar{C}(\mathfrak{M})$ such that $(f g)(x)=f(x) g(x)$ and $(f+g)(x)=f(x)+g(x)$, respectively, except on a set of the first category (cf. [5] and [10]).) Let $E(\sigma)$ be the spectral measure of $R$. If $\hat{T} \in \bar{C}(\mathfrak{M})$, let $T$ be the normal operator (in general unbounded) $T=\int_{M} \hat{T}(M) d E(M) .\left(u \in D(T)\right.$ if and only if $\int_{M}|\hat{T}(M)|^{2} d\|E(M) u\|^{2}<\infty$.) Let $\bar{R}$ be the set of all normal operators $\{T \mid \hat{T} \in \bar{C}(\mathfrak{M})\}$ and define the sum and product of any two operators $T$ and $S$ in $\bar{R}$ to be $[T+S]$ and [TS], respectively. $\bar{R}$ together with these operations and the usual operations of multiplications by scalars and adjunction is a commutative $*$-algebra and the mapping $\hat{T} \rightarrow T$ is a $*$-isomorphism of $\bar{C}(\mathfrak{M})$ onto $\bar{R}$ (cf. loc. cit.). Now, $T_{x} \in \bar{R}$ for all $x \in A_{e}$ (the proof is the same as that of Theorem 4 in [10]), and hence $x \rightarrow \hat{T}_{x}$ is a $*$-homomorphism of $A_{e}$ into $\bar{C}(\mathfrak{M})$ and

$$
f\left(x y z^{*}\right)=\left(T_{x} y_{f} \mid z_{f}\right)=\int_{\mathfrak{M}} \hat{T}_{x}(M) d\left(E(M) y_{f} \mid z_{f}\right)
$$

for all $x, y$, and $z$ in $A$. We denote by $\mu_{x}$, if $x \in A$, the Radon measure which for every Borel set $\sigma \subset \mathfrak{M}$ is defined by $\mu_{x}(\sigma)=\left\|E(\sigma) x_{f}\right\|^{2}$. If $x \in A_{e}$, let $S_{x}$ be the set of all $M$ such that $\left|\hat{T}_{x}(M)\right|=\infty$. $S_{x}$ is nowhere dense and hence $E\left(S_{x}\right)=0$ (for $\hat{E}\left(S_{x}\right)$ 
is the characteristic function of $\varnothing$ (cf. [10, p. 134])) and therefore $\mu_{y}\left(S_{x}\right)=0$ for all $y \in A$. Therefore $\hat{T}_{x}$ is finite $\mu_{y}$-a.e. for every $y \in A$. Now, for every $x$ and $y$ in $A$ and $\hat{T} \in C(\mathfrak{M})$,

$$
\begin{aligned}
\int_{\mathfrak{M}} \hat{T}(M)\left|\hat{T}_{y}(M)\right|^{2} d \mu_{x}(M) & =\left(T T_{y} T_{y}^{*} x_{f} \mid x_{f}\right) \\
& =\left(T T_{x} T_{x}^{*} y_{f} \mid y_{f}\right)=\int_{\mathfrak{M}} \hat{T}(M)\left|T_{x}(M)\right|^{2} d \mu_{y}(M)
\end{aligned}
$$

and hence $\left|\hat{T}_{y}(M)\right|^{2} d \mu_{x}(M)=\left|\hat{T}_{x}(M)\right|^{2} d \mu_{y}(M)$ for all $x$ and $y$ in $A$.

Let $X$ be the set of $M \in \mathfrak{M}$ such that $\hat{T}_{x}(M) \neq 0$ for some $x \in A$. $X$ is an open subset of $\mathfrak{M}$ and hence locally compact. Let $\nu_{x}$ be the restriction of the Radon measure $\mu_{x}$ to $X$ and denote the restriction of a function $\hat{T} \in \bar{C}(\mathfrak{M})$ to $X$ by $\tilde{T}$. (In this note we follow Bourbaki's approach to measure theory [1], [2].) Then $\left|\tilde{T}_{x}(M)\right|^{2} d \nu_{y}(M)=\left|\tilde{T}_{y}(M)\right|^{2} d \nu_{x}(M)$ for all $x$ and $y$ in $A$.

TheOREM 3. There exists a positive Radon measure $\nu$ on $X$ such that $\tilde{T}_{x} \in L^{2}(\nu)$ and $d \nu_{x}(M)=\left|\tilde{T}_{x}(M)\right|^{2} d \nu(M)$ for all $x \in A$ and $f\left(x y z^{*}\right)=\int_{X} \tilde{T}_{x y z^{*}}(M) d \nu(M)$ for all $x, y$, and $z$ in $A$.

Proof. If $K$ is a compact set in $X$, then there exists an $x \in A$ such that $\tilde{T}_{x}(M) \neq 0$ for all $M \in K$. Indeed, if $M \in K$, there exists an element $y=y_{M} \in A$ such that $\tilde{T}_{y}(M) \neq 0$. Hence there exists an open neighborhood $U_{y}$ of $M$ on which $\tilde{T}_{y}$ does not vanish. Since $K$ is compact, there exist finitely many such $U_{y}: U_{y_{i}}, i=1,2, \ldots, n$, such that $K \subset \bigcup_{i=1}^{n} U_{y_{i}}$. Let $x=y_{1} y_{1}^{*}+y_{2} y_{2}^{*}+\cdots+y_{n} y_{n}^{*}$, then $\tilde{T}_{x}(M)=\left|\tilde{T}_{y_{1}}(M)\right|^{2}$ $+\left|\tilde{T}_{y_{2}}(M)\right|^{2}+\cdots+\left|\tilde{T}_{y_{n}}(M)\right|^{2}$ for all $M \in X$ (equality holds for all $M$ because the sum of the right-hand side is everywhere continuous) and hence $\tilde{T}_{x}(M)>0$ on $K$.

Let $C_{00}(X)$ be the vector space of complex-valued continuous functions on $X$ with compact support. If $\varphi \in C_{00}(X)$ and $\sigma_{\varphi}$ is the support of $\varphi$, we choose an element $x \in A$ such that $\tilde{T}_{x}(M) \neq 0$ on $\sigma_{\varphi}$. Then $\varphi /\left|\tilde{T}_{x}\right|^{2} \in C_{00}(X)\left(\varphi /\left|\tilde{T}_{x}\right|^{2}\right.$ denotes the function which is equal to $\varphi(M) /\left|\tilde{T}_{x}(M)\right|^{2}$ for $M \in \sigma_{\varphi}$ and 0 for $\left.M \notin \sigma_{\varphi}\right)$ and set $\nu(\varphi)=\int\left(\varphi /\left|\tilde{T}_{x}\right|^{2}\right) d \nu_{x}$. The definition of $\nu$ is independent of the particular choice of $x$, for if $y$ is another element in $A$ such that $\tilde{T}_{y}(M) \neq 0$ on $\sigma_{\varphi}$, then

$$
\begin{aligned}
\int \frac{\varphi}{\left|\tilde{T}_{x}\right|^{2}} d \nu_{x} & =\int_{\sigma_{\varphi}} \frac{\varphi(M)}{\left|\tilde{T}_{x}(M)\right|^{2}} d \nu_{x}(M)=\int_{\sigma_{\varphi}} \frac{\varphi(M)}{\left|\tilde{T}_{y}(M)\right|^{2}} \frac{\left|\tilde{T}_{y}(M)\right|^{2}}{\left|\tilde{T}_{x}(M)\right|^{2}} d \nu_{x}(M) \\
& =\int_{\sigma_{\varphi}} \frac{\varphi(M)}{\left|\tilde{T}_{y}(M)\right|^{2}} \frac{\left|\tilde{T}_{x}(M)\right|^{2}}{\left|\tilde{T}_{x}(M)\right|^{2}} d \nu_{y}(M)=\int_{\sigma_{\varphi}} \frac{\varphi(M)}{\left|\tilde{T}_{y}(M)\right|^{2}} d \nu_{y}(M)=\int \frac{\varphi}{\left|\tilde{T}_{y}\right|^{2}} d \nu_{y} .
\end{aligned}
$$

Now, $\nu(\varphi) \geqq 0$ if $\varphi \geqq 0$ and hence $\nu$ is a positive Radon measure on $X$.

Let $N_{x}=\left\{M \in X \mid \tilde{T}_{x}(M)=0\right\}$, then $\nu_{x}\left(N_{x}\right)=0$. Indeed, if $C$ is a compact subset of $N_{x}$ choose $y \in A$ such that $\tilde{T}_{y}(M) \neq 0$ on $C$. Then

$$
\int_{C}\left|\tilde{T}_{y}(M)\right|^{2} d \nu_{x}(M)=\int_{C}\left|\tilde{T}_{x}(M)\right|^{2} d \nu_{y}(M)=0
$$


and therefore $\nu_{x}(C)=0$. Hence $\nu_{x}\left(N_{x}\right)=0$. We assert also that $\nu\left(S_{x}\right)=0$. Indeed, for every integer $n>0$ let $G_{n}=\left\{M \in X|| \tilde{T}_{x}(M) \mid>n\right\}$, then $\bar{G}_{n}$ (closure of $G_{n}$ in $X$ ) is clopen and compact and hence

Therefore $\nu\left(S_{x}\right)=0$.

$$
\nu\left(S_{x}\right) \leqq \nu\left(\bar{G}_{n}\right)=\int_{\bar{G}_{n}} \frac{d \nu_{x}(M)}{\left|\widetilde{T}_{x}(M)\right|^{2}} \leqq \frac{f\left(x x^{*}\right)}{n^{2}} .
$$

Let $\varphi \in C_{00}^{+}(X)$ (nonnegative real-valued elements of $C_{00}(X)$ ) and $x \in A$. For every integer $n>0$ let $\sigma_{n}=\left\{M \in X|1 / n<| \tilde{T}_{x}(M) \mid<n\right\}$. $\bar{\sigma}_{n}$ (closure of $\sigma_{n}$ in $X$ ) is clopen and contained in $\left\{M \in X|1 / n \leqq| \tilde{T}_{x}(M) \mid \leqq n\right\}$ and therefore is compact. Hence

$$
\int_{\tilde{\sigma}_{n}} \varphi(M) d \nu_{x}(M)=\int_{\tilde{\sigma}_{n}} \varphi(M) \frac{\left|\tilde{T}_{x}(M)\right|^{2}}{\left|\tilde{T}_{x}(M)\right|^{2}} d \nu_{x}(M)=\int_{\tilde{\sigma}_{n}} \varphi(M)\left|\tilde{T}_{x}(M)\right|^{2} d \nu(M) .
$$

Letting $n \rightarrow \infty$ we obtain

$$
\int_{X-\left(S_{x} \cup N_{x}\right)} \varphi(M) d \nu_{x}(M)=\int_{X-\left(S_{x} \cup N_{x}\right)} \varphi(M)\left|\tilde{T}_{x}(M)\right|^{2} d \nu(M)
$$

by the monotone convergence theorem. But $v_{x}\left(N_{x}\right)=v_{x}\left(S_{x}\right)=\nu\left(S_{x}\right)=0$ as we have seen. Hence

$$
\int_{X} \varphi(M) d \nu_{x}(M)=\int_{X} \varphi(M)\left|\tilde{T}_{x}(M)\right|^{2} d \nu(M)
$$

Therefore $\int_{X} \varphi(M) d \nu_{x}(M)=\int_{X} \varphi(M)\left|\tilde{T}_{x}(M)\right|^{2} d \nu(M)$ for all $\varphi \in C_{00}(X)$; that is, $d \nu_{x}(M)=\left|\tilde{T}_{x}(M)\right|^{2} d \nu(M)$ (and therefore $\tilde{T}_{x} \in L^{2}(\nu)$ ).

Finally, let $x$ and $y$ be arbitrary elements in $A$ and $\Delta_{n}=\left\{M \in X|1 / n<| \tilde{T}_{x}(M) \mid<n\right.$, $\left.1 / n<\left|\tilde{T}_{y}(M)\right|<n\right\}$. Then $\bar{\Delta}_{n}$ is clopen and compact and

$$
\int_{\bar{\Delta}_{n}} \tilde{T}_{x}(M) d \nu_{y}(M)=\int_{\bar{\Delta}_{n}} \tilde{T}_{x}(M)\left|\tilde{T}_{y}(M)\right|^{2} d \nu(M)=\int_{\bar{\Delta}_{n}} \tilde{T}_{x y y^{*}}(M) d \nu(M) .
$$

Now $\tilde{T}_{x} \in L^{1}\left(\nu_{y}\right)$ and $\tilde{T}_{x y y^{*}} \in L^{1}(\nu)$ since $\tilde{T}_{x}$ and $\tilde{T}_{y y^{*}}$ belong to $L^{2}(\nu)$. Hence, letting $n \rightarrow \infty$ we obtain

$$
\int_{X} \tilde{T}_{x}(M) d \nu_{y}(M)=\int_{X} \tilde{T}_{x y y *}(M) d \nu(M)
$$

by Lebesgue's dominated convergence theorem and the fact that $\nu\left(S_{x}\right)=\nu\left(S_{y}\right)$ $=\nu_{y}\left(N_{y}\right)=\nu_{y}\left(S_{y}\right)=0$. But $f\left(x y y^{*}\right)=\int_{X} \tilde{T}_{x}(M) d \nu_{y}(M)$. Therefore

$$
f\left(x y y^{*}\right)=\int_{X} \tilde{T}_{x y y^{*}}(M) d \nu(M)
$$

and hence, using the identity

$$
\begin{array}{r}
f\left(x y z^{*}\right)=\frac{1}{4}\left\{f\left(x(y+z)(y+z)^{*}\right)-f\left(x(y-z)(y-z)^{*}\right)+i f\left(x(y+i z)(y+i z)^{*}\right)\right. \\
\left.-i f\left(x(y-i z)(y-i z)^{*}\right)\right\}
\end{array}
$$


we obtain

$$
f\left(x y z^{*}\right)=\int_{X} \tilde{T}_{x y z^{*}}(M) d \nu(M) \text { for all } x, y, \text { and } z \text { in } A .
$$

COROLlaRY 1. If $N$ is a v-measurable subset of $X$ such that $\nu_{x}(N)=0$ for all $x \in A$, then $N$ is $\nu$-locally negligible.

Proof. We first observe that if $N$ is a $\nu$-measurable set, then $N$ is $\nu_{x}$-measurable, because $N-N_{x}$ is $\nu$-measurable (since $N_{x}$ is closed) (cf. [1, p. 43]). Let $C$ be a compact subset of $X$ and $N$ a $\nu$-measurable set such that $\nu_{x}(N)=0$ for all $x \in A$. Choose an element $y \in A$ such that $\tilde{T}_{y}(M) \neq 0$ on $C$. Then

$$
0=\nu_{y}(C \cap N)=\int_{C \cap N}\left|\tilde{T}_{y}(M)\right|^{2} d \nu(M)
$$

and therefore $\nu(C \cap N)=0$.

Let $S=\bigcup_{x \in A} S_{x}$, where $S_{x}$ is as above the set of $M \in X$ such that $\left|\tilde{T}_{x}(M)\right|=\infty$. We shall give a sufficient condition for $\bar{S}$ to be $\nu$-locally negligible.

LEMMA 2. If $f$ satisfies the additional condition (d): there exists a countable subset $D$ of $A_{e}$ such that for every $x \in A$ there exists a $y \in A_{e}$ which is a polynomial with complex coefficients in finitely many elements of $D$ such that

$$
f\left(x x^{*} z z^{*}\right) \leqq f\left(y y^{*} z z^{*}\right) \text { for all } z \in A,
$$

then $\bar{S}$ is v-locally negligible.

Proof. We shall show that if $f$ satisfies condition (d), then $S=\bigcup_{x \in D} S_{x}$. Indeed, let $x$ be an arbitrary element in $A$ and $y$ be an element in $A_{e}$ which is a polynomial in finitely many elements of $D$ such that $f\left(x x^{*} z z^{*}\right) \leqq f\left(y y^{*} z z^{*}\right)$ for all $z \in A$. This inequality is equivalent with the inequality $\left\|T_{x} u\right\| \leqq\left\|T_{y} u\right\|$ for all $u \in H_{f}^{\prime}$. This implies that $D\left(T_{y}\right) \subset D\left(T_{x}\right)$ and $\left\|T_{x} u\right\| \leqq\left\|T_{y} u\right\|$ for all $u \in D\left(T_{y}\right)$, since $T_{y}$ and $T_{x}$ are the closures of their restrictions, respectively, to $H_{f}^{\prime}$. This implies in turn that $\left|\hat{T}_{x}(M)\right| \leqq\left|\hat{T}_{y}(M)\right|$ for all $M \in \mathfrak{M}$. Indeed, suppose that $\left|\hat{T}_{x}\left(M_{0}\right)\right|>\left|\hat{T}_{y}\left(M_{0}\right)\right|$. Then $\left|\hat{T}_{y}\left(M_{0}\right)\right|<\infty$ and hence there exists a clopen neighborhood $\sigma$ of $M_{0}$ and a positive number $\varepsilon$ such that $\left|\hat{T}_{x}(M)\right|^{2}>\left|\hat{T}_{y}(M)\right|^{2}+\varepsilon$ for all $M \in \sigma . E(\sigma) \neq 0$ since $\sigma \neq \varnothing$. We may therefore choose a nonzero vector $u$ in the range of $E(\sigma)$ and since $\hat{T}_{y}$ is bounded on $\sigma$ it follows that $u \in D\left(T_{y}\right) \subset D\left(T_{x}\right)$ and hence

$$
\begin{aligned}
\left\|T_{x} u\right\|^{2} & =\int_{\mathfrak{M}}\left|\hat{T}_{x}(M)\right|^{2} d\|E(M) u\|^{2}=\int_{\sigma}\left|\hat{T}_{x}(M)\right|^{2} d\|E(M) u\|^{2} \\
& \geqq \int_{\sigma}\left(\left|\hat{T}_{y}(M)\right|^{2}+\varepsilon\right) d\|E(M) u\|^{2}=\left\|T_{y} u\right\|^{2}+\varepsilon\|u\|^{2} .
\end{aligned}
$$

This is a contradiction. From the fact that $\left|\hat{T}_{x}\right| \leqq\left|\hat{T}_{y}\right|$ follows that $S_{x} \subset S_{y}$. But clearly $S_{y} \subset \bigcup_{z \in D} S_{z}\left({ }^{2}\right)$. Hence $S \subset \bigcup_{z \in D} S_{z}$ and therefore $S=\bigcup_{z \in D} S_{z}$.

(2) For $S_{x+y} \subset S_{x} \cup S_{y}$ and $S_{x y} \subset S_{x} \cup S_{y}$ (cf. [10, p. 136]). 
Since $D$ is a countable set and every $S_{z}$ is nowhere dense, it follows that $S$ is a set of the first category in $\mathfrak{M}$. But in $\mathfrak{M}$ every set of the first category is nowhere dense (cf. [10] or [1, p. 65]). Hence $S$ is nowhere dense in $\mathfrak{M}$ and therefore the closure $\bar{S}^{\mathfrak{M}}$ of $S$ in $\mathfrak{M}$ is nowhere dense in $\mathfrak{M}$. Hence $\mu_{x}\left(\bar{S}^{\mathfrak{M}}\right)=0$ for all $x \in A$ (for $\left.E\left(\bar{S}^{\mathfrak{M}}\right)=0\right)$. Hence $\nu_{x}(\bar{S})=0$ for all $x \in A(\bar{S}$ denotes the closure of $S$ in $X)$ and therefore $\bar{S}$ is $\nu$-locally negligible by Corollary 1 .

REMARK. If $f$ is unitary we know a priori that $S=\varnothing$, since the operators $T_{x}$ are bounded in that case. But $f$ clearly satisfies also condition (d) (cf. Introduction).

We are now ready to prove the main theorem which is an extension of Theorem 1 of R. Godement.

THEOREM 4. Let $f$ be a positive linear functional on a commutative *-algebra A. If $f$ is quasi-unitary and satisfies condition (d), then there exists a positive Radon measure $\mu_{f}$ on a locally compact subset $\sigma_{f}$ of $\hat{A}$ such that

(a) $\hat{x}(\chi)=\chi(x)$ belongs to $L^{2}\left(\mu_{f}\right)$ for every $x \in A$;

(b) $f(x y z)=\int_{\sigma_{f}} \chi(x y z) d \mu_{f}(\chi)$ for all $x, y$, and $z$ in $A$.

If, furthermore, $f$ is extendible, then $\mu_{f}$ is a finite measure and

for all $x$ in $A$.

$$
f(x)=\int_{\sigma_{f}} \chi(x) d \mu_{f}(\chi)
$$

Proof. By Theorem 3 and Lemma 2

$$
f(x y z)=\int_{X^{\prime}} T_{x y z}^{\prime}(M) d \nu^{\prime}(M) \text { for all } x, y, \text { and } z \text { in } A,
$$

where $X^{\prime}=X-\bar{S}, T_{x}^{\prime}$ is the restriction of $\tilde{T}_{x}$ to $X^{\prime}$ and $\nu^{\prime}$ is the restriction of the Radon measure $\nu$ to $X^{\prime}$ ( $X^{\prime}$ is an open subset of $X$ and hence locally compact). The mapping $x \rightarrow T_{x}^{\prime}(M)$ is a unitary character of $A$ for every $M \in X^{\prime}$, since $x \rightarrow \hat{T}_{x}$ is a $*$-homomorphism of $A$ into $\bar{C}(\mathfrak{M})$. Let $\varphi$ be the mapping of $X^{\prime}$ into $\hat{A}$ which maps $M$ into $T_{(\cdot)}^{\prime}(M) . \varphi$ is continuous because $M \rightarrow T_{x}^{\prime}(M)$ is a continuous mapping on $X^{\prime}$ for every fixed $x \in A$.

Let $\sigma_{f}=\varphi\left(X^{\prime}\right) . \sigma_{f}$ is locally compact, for if $\varphi\left(M_{0}\right)=T_{(\cdot)}^{\prime}\left(M_{0}\right) \in \sigma_{f}$, let $x_{0}$ be an element of $A$ such that $T_{x_{0}}^{\prime}\left(M_{0}\right) \neq 0, \varepsilon=\left|T_{x_{0}}^{\prime}\left(M_{0}\right)\right| / 2$ and

$$
\hat{N}=\left\{\chi \in \sigma_{f}|| \chi\left(x_{0}\right)-T_{x_{0}}^{\prime}\left(M_{0}\right) \mid \leqq \varepsilon\right\} .
$$

$\hat{N}$ is clearly a neighborhood of $\varphi\left(M_{0}\right)$ and

$$
N=\varphi^{-1}(\hat{N})=\left\{M \in X^{\prime}|| T_{x_{0}}^{\prime}(M)-T_{x_{0}}^{\prime}\left(M_{0}\right) \mid \leqq \varepsilon\right\} .
$$

$N$ is a compact neighborhood of $M_{0}$ for $\left\{M \in \mathfrak{M}|| \hat{T}_{x_{0}}(M)-\hat{T}_{x_{0}}\left(M_{0}\right) \mid \leqq \varepsilon\right\}$ is a compact neighborhood of $M_{0}$ in $\mathfrak{M}$ and $N=\left\{M \in \mathfrak{M}|| \hat{T}_{x_{0}}(M)-\hat{T}_{x_{0}}\left(M_{0}\right) \mid \leqq \varepsilon\right\}$. Since $\varphi$ is continuous and $\hat{N}=\varphi(N)$, it follows that $\hat{N}$ is compact.

Next, we show that $\varphi$ is a proper mapping; that is, if $C$ is a compact set in $\sigma_{f}$, then $\varphi^{-1}(C)$ is a compact set in $X^{\prime}$. Let $C$ be a compact set in $\sigma_{f}$ and $K=\varphi^{-1}(C)$. 
Since $C$ is compact, there exists by what precedes a finite number of compact neighborhoods $\hat{N}_{1}, \hat{N}_{2}, \ldots, \hat{N}_{n}$ of points in $C$ such that $\varphi^{-1}\left(\hat{N}_{i}\right)=N_{i}$ is compact for $i=1,2, \ldots, n$, and $C \subset \bigcup_{i=1}^{n} \hat{N}_{i}$. Hence $K \subset \bigcup_{i=1}^{n} N_{i}$. Since $K$ is closed and $\bigcup_{i=1}^{n} N_{i}$ is compact, it follows that $K$ is compact.

Let now $\mu_{f}$ be the image of the Radon measure $\nu^{\prime}$ under $\varphi$ (i.e. $\mu_{f}$ is the Radon measure on $\sigma_{f}$ defined by $\int_{\sigma_{f}} g d \mu_{f}=\int_{X^{\prime}}(g \circ \varphi) d \nu^{\prime}$ for all $\left.g \in C_{00}\left(\sigma_{f}\right)\right)$ then

$$
f(x y z)=\int_{\sigma_{f}} \chi(x y z) d \mu_{f}(\chi) \text { for all } x, y, \text { and } z \text { in } A \text {. }
$$

That $\hat{x}(\chi)=\chi(x)$ belongs to $L^{2}\left(\mu_{f}\right)$ follows from the fact that $T_{x}^{\prime} \in L^{2}\left(\nu^{\prime}\right)$ by Theorem 3 for every $x \in A$.

Finally, if $f$ is extendible, let $f$ be the positive linear functional on $A_{e}$ which extends $f$. We may assume that $f \neq 0$, for otherwise the assertion of the theorem is trivially true. It is easily seen that $\tilde{f}$ is quasi-unitary. Let $x \rightarrow T_{x}$ be the $*$-representation of $A_{e}$ corresponding to $f^{f}$ (cf. Theorem 2). Then

$$
f(x)=\tilde{f}(x)=\left(T_{x} e_{\tilde{f}} \mid e_{\tilde{f}}\right)=\int_{\mathfrak{M}} \hat{T}_{x}(M) d \mu(M)
$$

for all $x \in A$, where $\mu(\sigma)=\left\|E(\sigma) e_{\tilde{f}}\right\|^{2}$. Clearly $\mu$ is a bounded measure and $\hat{T}_{x} \in L^{2}(\mu)$ for all $x \in A$. It is easily seen that $\tilde{f}$ satisfies condition (d) and hence $\bar{S}$ is $\mu$-locally negligible. Let $X$ be the set of all $M$ in $\mathfrak{M}$ such that $\hat{T}_{x}(M) \neq 0$ for some $x \in A$. $X$ is an open subset of $\mathfrak{M}$ and hence $X^{\prime}=X-\bar{S}$ is an open subset of $\mathfrak{M}$ and therefore locally compact. Let-using the same notation as above $-T_{x}^{\prime}$ be the restriction of $\hat{T}_{x}$ to $X^{\prime}$ and $\nu^{\prime}$ the restriction of the Radon measure $\mu$ to $X^{\prime}$, then

$$
f(x)=\int_{X^{\prime}} T_{x}^{\prime}(M) d \nu^{\prime}(M) \text { for all } x \in A .
$$

Let as above $\varphi$ be the mapping of $X^{\prime}$ into $\hat{A}$ which maps $M$ into $T_{(\cdot)}^{\prime}(M)$. The rest of the proof is identical with the preceding argument and we obtain the formula

$$
f(x)=\int_{\sigma_{f}} \chi(x) d \mu_{f}(\chi) \text { for all } x \in A,
$$

where $\mu_{f}$ is a bounded measure on $\sigma_{f}$ (as the image under $\varphi$ of the bounded measure $\left.\nu^{\prime}\right)$.

REMARK: If $f$ is unitary, then the functions $\hat{x}(\chi)=\chi(x)$ are bounded on $\sigma_{f}$. In fact, in that case $|\hat{x}(\chi)| \leqq M_{x}$ for all $\chi \in \sigma_{f}$. If $f$ is not unitary but quasi-unitary and satisfies condition (d), then the functions $\hat{x}$ are not in general bounded on $\sigma_{f}$.

\section{REFERENCES}

1. N. Bourbaki, Intégration des mesures, Hermann, Paris, 1956.

2. - Intégration, Hermann, Paris, 1952.

3. T. Carleman, Les fonctions quasi-analytiques, Gauthier-Villars, Paris, 1926.

4. J. Dixmier, Sur certains espaces considérés par M. H. Stone, Summa Brasil. Math. 2 (1951), 151-182. 
5. J. M. G. Fell and J. L. Kelley, An algebra of unbounded operators, Proc. Nat. Acad. Sci. U.S.A. 38 (1952), 592-598.

6. R. Godement, Sur la théorie des représentations unitaires, Ann. of Math. (2) 53 (1951), 68-124.

7. I. M. Gelfand and N. Ya. Vilenkin, Generalized functions, Vol. 4, Academic Press, New York, 1964.

8. L. H. Loomis, An Introduction to abstract harmonic analysis, Van Nostrand, New York, 1953.

9. M. A. Naimark, Normed rings, Noordhoff, Groningen, 1959.

10. A. E. Nussbaum, Integral representation of semi-groups of unbounded self-adjoint operators, Ann. of Math. (2) 69 (1959), 133-141.

11. - Quasi-analytic vectors, Ark. Mat. 6 (1965), 179-191.

12. C. E. Rickart, Banach algebras, Van Nostrand, New York, 1960.

13. R. B. Zarhina, On the two-dimensional problem of moments, Dokl. Akad. Nauk. SSSR 124 (1959), 743-746. (Russian)

WASHINGTON UNIVERSITY,

ST. LouIS, Missouri 